\title{
La Segmentación De Mercado Y La Satisfacción Del Cliente En Los Negocios Comerciales De La Bahía De Guayaquil
}

\author{
Econ. Rosa Espinoza Toalombo (MAE) \\ Est. Jenniffer Alchundia \\ Est. Xavier Layana \\ Ing. Xiomara Zuñiga (MAE) \\ Ing. Diego Tapia (MAE) \\ Universidad Estatal de Milagro, Ecuador \\ Lcdo. Wilmer Espinoza (MT)
}

Universidad Laica Vicente Rocafuerte de Guayquil, Ecuador

Doi:10.19044/esj.2018.v14n25p126 URL:http://dx.doi.org/10.19044/esj.2018.v14n25p126

\begin{abstract}
Currently, majority of people who make up the society have a lot of different needs that the market must be willing to meet. The application of the segmentation strategy allows the offering of a commercial mix for the different types of consumers, giving the opportunity to customize and structure a unique combination of elements such as product, price, place, and promotion. This value proposition is potentially superior to that of competition. As a result, this is why it is imperative to study the segmentation of the market and the impact that this gives to the satisfaction of customers who like the goods and services offered in the Bay of Guayaquil. It, however, focuses on contributing to the improvement of the structure and positioning of these markets. The methodology used is empirical-positivist because it generates a new knowledge. The field is in relation to the sample that was surveyed. This study is exploratory because they intend to give us an overview regarding a reality. Finally, the study is descriptive because it details the results of the study. The adaptation of market segmentation is a feasible strategy to meet the needs of consumers and mitigate the risks that the markets present. As a result, it is advisable to divide it by groups in order to differentiate consumers and respond more safely to their preferential needs.
\end{abstract}

Keywords: Segmentation, Market, Consumer, Marketing, Strategy 


\section{Resumen}

Actualmente las personas que conforman una sociedad tienen necesidades muy diversas, razón por la cual el mercado debe estar dispuesto a satisfacerlas. La aplicación de la estrategia de segmentación permite ofrecer una mezcla comercial para los distintos tipos de consumidores, dando la oportunidad de personalizar y estructurar a la medida una combinación única de elementos como el producto, el precio, la plaza y la promoción. Esta propuesta de valor es potencialmente superior a la de la competencia, por lo cual es imprescindible estudiar la segmentación de mercado y el impacto que este otorga a la satisfacción de los clientes que disfrutan de los bienes y servicios que se ofertan en la Bahía de Guayaquil, con el fin de contribuir al mejoramiento de la estructura y posicionamiento de dichos mercados. La metodología empleada es empírica- positivista, pues genera un nuevo conocimiento de campo en relación a la encuesta, exploratoria debido a que pretende darnos una visión general respecto a una realidad y descriptiva porque detalla los resultados del estudio. La adaptación de la segmentación de mercado resulta una estrategia factible para satisfacer las necesidades de los consumidores y mitigar los riesgos que los mercados presentan, por ende es conveniente dividirlo por grupos para poder diferenciar a los consumidores y responder con mayor seguridad a sus necesidades preferenciales.

Palabras claves: Segmentación, Mercado, Consumidor, Marketing, Estrategias

\section{Introducción}

El Estudio de la segmentación de mercado en los negocios comerciales de la Bahía permite comprender parte de la realidad de los mercados populares de la ciudad de Guayaquil. Dicha realidad muestra las falencias que aún existen sobre estos mercados que llevan por ende a la insatisfacción de los clientes, demostrando que aún queda un gran trabajo por realizar en esta área del marketing.

La segmentación de mercados es uno de los temas más básicos al hablar de mercadeo que no sólo menciona los aspectos geográficos, psicográficos y conductuales, sino que también toma en cuenta la creación de un grupo objetivo para un producto en específico. Sin embargo, en la práctica muchas veces se pasa por alto este punto que es vital para el buen desarrollo de las estrategias; es decir, no se trata de sentarse a definir una gran cantidad de características similares de un grupo en específico, en realidad se trata de ser consistentes con un mismo segmento y sobre todo lograr un posicionamiento claro, fuerte y positivo dentro del mercado de interés (Cabrera, 2016). La definición que ha propuesto anteriormente no se aleja mucho de lo afirmado hace años por Smith, pues de alguna forma se relaciona a lo que hoy en día es 
evidente en los mercados de bienes y servicios. En el marketing el mercado puede ser un espacio tanto físico como virtual que se caracteriza por ser heterogéneo, pero que dentro de sí contiene un número significativo de mercados pequeños homogéneos dispuestos a complacer las diferentes necesidades del consumidor, así como también sus deseos desde los más sencillos hasta los más sofisticados.

Se justifica esta investigación por la necesidad de contribuir al mejoramiento de la estructura y posicionamiento de los mercados de la Bahía incrementando así la competitividad con respecto a otros mercados nacionales. Guayaquil es una de las dos ciudades más grandes, tanto en lo territorial, como económico, aportando a la economía del país mediante el constante movimiento del comercio en los sectores como la Bahía. Es así que la idea que se pretende plasmar es de dar a conocer la estrategia de segmentación con un modelo efectivo para generar mayor satisfacción a los clientes mediante la creación de mercados homogéneos dentro de una gama de productos y servicios heterogéneos, de forma que se puedan ofertar de una manera innovadora y poco utilizada en nuestro país.

De esta formasurge la necesidad de que el concepto de segmentación de mercado y estrategias de segmentación sean perfeccionados para poder llegar de una forma más directa a los consumidores que cada vez se vuelven más exigentes en sus gustos y requerimientos de mercado. Se plantea como objetivo de investigación dar a conocer de forma efectiva que la segmentación de mercado y el impacto que este otorga a la satisfacción de los clientes que disfrutan de los bienes y servicios que se ofertan en la Bahía de Guayaquil. Para alcanzar estos objetivos también se han planteado los siguientes objetivos específicos:

- Clasificar las variables que permiten generar una segmentación de mercado eficaz y efectiva.

- Determinar los factores que impiden la aplicación de una segmentación de mercado como estrategia de marketing.

\section{Hipótesis}

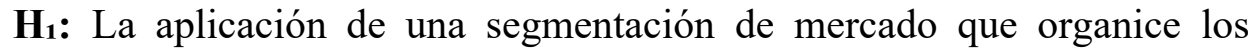
negocios de la bahía de Guayaquil genera la variable de satisfacción en los clientes a la hora de comprar.

$\mathbf{H}_{2}$ : La aplicación de la segmentación de mercado en los negocios de la bahía de Guayaquil se debe a diversos factores que influyen en la actitud del ofertante al momento de implementarla. 


\section{Aspectos conceptuales del marketing, segmentación de mercado y satisfacción del cliente}

El nacimiento del marketing crea polémica en cuanto a una fecha y lugar específico de procedencia, por tal razón los autores no logran ponerse de acuerdo en este punto. Sin embargo, muchos sostienen que el Marketing es una actividad que se viene ejerciendo desde lo antiguo de la humanidad, pues el ser humano desde años ha estado involucrado con compra y venta de bienes y servicios. En palabras sencillas, el hombre siempre ha estado ligado al trueque y con el tiempo ha evolucionado sus formas de intercambios con el fin de satisfacer sus necesidades (Arrieta, 2007). Definiéndolo en términos sencillos, el marketing es un proceso en el cual los individuos y empresas obtienen lo que necesitan; es decir, intercambian valor con otros, estableciendo relaciones redituables, intercambio de valor, con los clientes (Kotler \& Armstrong, 2012).

El concepto de mercadotecnia con el tiempo ha evolucionado al punto que hoy en día es un aspecto imprescindible del negocio en relación al mercado. En este sentido, el mercado se compone por consumidores que se encuentran en un lugar determinado, en el cual hay factores geográficos, sociales, económicos, entre otros. El conocimiento de cada uno de estos factores constituye un elemento fundamental para poner en marcha el marketing y sus estrategias (Circulos de Lectores, 2004).

El marketing como elemento esencial en los negocios al presentar un producto en el mercado, se vuelve una estrategia predominante de la mercadotecnia, calando muy alto entre las estrategias de marketing. Para lograr el correcto uso del marketing son necesarias fases como la planificación y ejecución apegadas a técnicas de última generación y sobre todo que sean creativas e innovadoras, pues el mercado normalmente es exigente y crítico (Kotler \& Armstrong, 2012). Como estrategia ha ayudado y permitido que muchos productos puedan posicionarse dentro de un mercado competitivo, así como ser aceptados con el fin de generar mejores bienes y servicios conforme mencionan los especialistas en marketing (Keller \& Kotler, 2012).

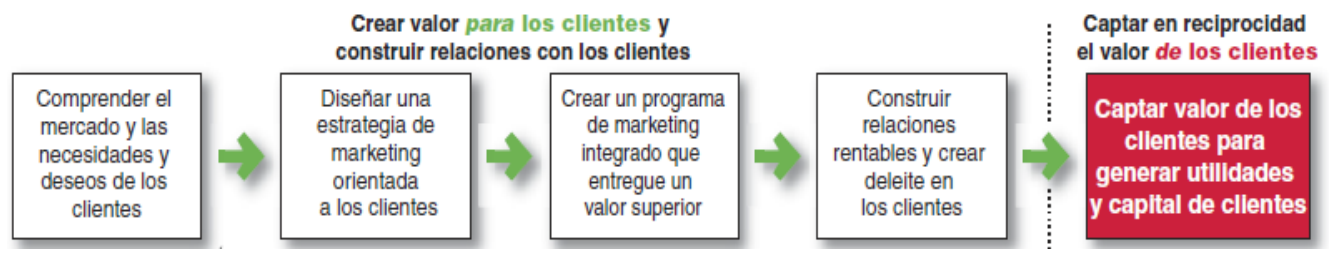

Figura 1. Un modelo sencillo del proceso de marketing Fuente: (Armstrong \& Kotler, 2013).

Se puede observar que la Figura 1. de Armstrong y Kotler nos muestra un modelo de 5 pasos del proceso que encierra el marketing, en donde el éxito 
de captar el valor de los clientes radica en la gestión que se realice mediante la doble meta del marketing; es decir, entender e identificar cuáles son las necesidades del mercado y sus clientes para saber qué ofertar. Luego, se debe armar la estrategia de marketing que trata de enganchar al cliente a través del ofrecimiento del producto o servicio. Para esto se requiere de un programa de marketing que indique paso a paso lo que se va a ejecutar con el fin de generar valor de intercambio y finalmente conseguir la lealtad del individuo, volviéndose un cliente potencial que generará futuras y constantes utilidades al negocio.

La segmentación de mercado desde sus comienzos fue abordada por (Smith, 1956) como un espacio de compra y venta homogéneo debido a la distribución de ciertos productos por segmentos determinados y que eran de preferencia del consumidor, de manera que se reunía a personas con gustos y preferencias similares. Con la división que promueve dicha segmentación de mercado cada consumidor logra obtener su grado de satisfacción con respecto al producto o servicio que está demandando, además de poder identificar eficaz y eficientemente las necesidades de los consumidores similares (Kotler \& Armstrong, 2008).

Con la segmentación de mercado se logra que la aplicación del marketing mix responda a los objetivos planteados en la misión de la empresa para con el mercado al que dirige su producto o servicio (Silk, 2006). Básicamente, la segmentación de mercado subdivide gran parte de los consumidores en pequeños segmentos con gustos, preferencias y necesidades similares. De dichos segmentos la empresa se nutre económicamente; es decir,sus clientes potenciales son los que aportan con flujo de dinero para el negocio (París, 2013).

Es fundamental que el gerente del negocio, a través de su departamento de marketing, elabore una estrategia que permita dividir un grupo de individuos con necesidades, circunstancias y características equivalentes y que respondan de la misma forma ante un producto o servicio. Después de ello hay que estudiar las necesidades del mercado y de cada uno de los segmentos previamente divididos, entre lo que están presentes las fortalezas, debilidades, oportunidades y amenazas de dicho mercado parafinalmente decidir sobre cómo lograr el posicionamiento del producto o servicio dentro de un mercado objetivo (Mullins, Walker, Boyd, \& Jamieson, 2013).

La estrategia de segmentar el mercado permite que los vendedores tengan una competencia mucho más regulada y frontal mediante la diferenciación en la oferta, precio y características del producto, mensajes publicitarios y método de distribución; es decir, diferenciación de ofertas en la mezcla de marketing (Robín \& Torres, 2001).

Dentro de la mezcla de marketing, la segmentación requiere de una correcta elección del mercado, así como del posicionamiento que logren las 
estrategias dirigidas al producto o servicio que trata de llamar la atención de los clientes. Es asícomo el proceso del marketing busca, a través de la creación e intercambio de valor, construir una relación de lealtad con el cliente y a cambio este le ofrece réditos fijos al negocio. En pocas palabras,a través de la satisfacción que el cliente obtenga por el producto o servicio este generará una ganancia constante (Armstrong \& Kotler, 2013).

\begin{tabular}{|c|c|c|}
\hline $\begin{array}{l}\text { Segmentación del mercado } \\
\text { Identificación de las bases } \\
\text { para la segmentación del } \\
\text { mercado } \\
\text { Desarrollo de perfiles } \\
\text { de segmentos }\end{array}$ & $\begin{array}{l}\quad \text { Marketing meta } \\
\text { Desarrollo de medidas } \\
\text { del atractivo del } \\
\text { segmento } \\
\text { Selección de los } \\
\text { segmentos meta }\end{array}$ & $\begin{array}{l}\text { Posicionamiento } \\
\text { en el mercado } \\
\text { Desarrollo de } \\
\text { posicionamiento para los } \\
\text { segmentos meta } \\
\text { Desarrollo de una mezcla } \\
\text { de marketing para cada } \\
\text { segmento }\end{array}$ \\
\hline
\end{tabular}

Figura 2. Pasos en la segmentación, búsqueda y posicionamiento del mercado meta

Fuente: (Kotler \& Armstrong, 2007)

El marketing en la actualidad según Kotler y Armstrong busca nuevas formas o estrategias para atraer a los compradores, los cuales son numerosos y con amplias necesidades de compra yademás las empresas, por su diversidad de productos y servicios, se direccionan a diferentes tipos de segmentos de mercado. Es por tal motivo que es necesario identificar qué parte del mercado es la indicada para obtener gananciasacción para la cual las estrategiasjueganun papel importante pues permiten desarrollar relaciones estrechas con los clientes.

Por lo general, las empresas al aplicar un marketing meta lo primero que deben desarrollar es la segmentación de mercado, pues les permite descubrir los gustos y preferencias de la sociedad haciéndolo por grupos homogéneos. Luego de aplicarse una efectiva segmentación de mercado se desarrollan las estrategias que permitan identificar el mercado meta mediante la evaluación individual de cada segmento para finalmente introducir el producto o servicio con el fin de posicionarlo, hacer que este sea superior que la competencia y que por ende se vuelva una necesidad en los clientes fijos y en los nuevos interesados en adquirirlo (Kotler \& Armstrong, 2007).

Para segmentar el mercado también existen diversos criterios, los cuales logran dividir el mercado de una forma ordenada y efectiva cumpliendo con las expectativas del consumidor, para lo cual se tienen segmentos por área geográfica, demográfica, psicográfica y comportamental. A continuación podremos observar cada uno de estos criterios (Ciribeli \& Miquelito, 2015). 
Tabla 1. Principales Criterios de Segmentación

\begin{tabular}{|l|l|}
\hline Geográfica & $\begin{array}{l}\text { El mercado es divido en unidades geográficas como país, estados, } \\
\text { ciudades, barros, clima, relieve, etc. }\end{array}$ \\
\hline Demográfica & $\begin{array}{l}\text { Los consumidores son clasificados llevando en consideración variables } \\
\text { como: edad, sexo, grado de instrucción, ingresos, estado civil, religión, } \\
\text { profesión, etc. }\end{array}$ \\
\hline Psicográfica & $\begin{array}{l}\text { El consumidor es clasificado de forma más subjetiva. En ese caso, son } \\
\text { relevantes los factores como: valores, actitudes, deseos, opiniones, } \\
\text { personalidad, estilo de vida. Personas que ocupan el mismo grupo } \\
\text { demográfico pueden tener perfiles psicográficos diferentes. }\end{array}$ \\
\hline Comportamental & $\begin{array}{l}\text { El mercado es segmentado de acuerdo con las características de } \\
\text { comportamiento del consumidor ante la compra. Son observados: } \\
\text { ocasión de la compra, frecuencia de la compra, fidelidad de consumo, } \\
\text { grado de uso, modo de utilización. }\end{array}$ \\
\hline
\end{tabular}

Fuente: (Ciribeli \& Miquelito, 2015)

A la hora de segmentar, la organización deberá considerar los diversos criterios que se muestran en la Figura 3, pues el consumidor es exigente y desea satisfacer una necesidad. Además el abordar una segmentación según los criterios planteados permite una mejor organización de los negocios y sobre todo de las preferencias que el cliente demanda del producto o servicio.

La satisfacción del cliente es muy importante en este aspecto del Marketing, pues de esto depende qué tan fijo se vuelve el cliente y qué cantidad ingresos genere a la empresa. Para lograr dicha aceptación la empresa debe implementar técnicas de comunicación y acercamiento con el cliente, para lo cual existen muchas formas de lograrlo pero siempre se deberá considerar los beneficios que se obtendrán, puesto que no pude ser mayor el valor de mantener al cliente que lo que éstos aporten a la empresa (Guadarrama \& Rosales, 2015).

La segmentación como estrategia en los negocios es sumamente importante, pues un esfuerzo de marketing bien planificado y dirigido a satisfacer las necesidades de un grupo de clientes semejantes es más poderoso y tiene más posibilidades de éxito (Stanton, Etze, \& Walker, 2007). Otro ejemplo de técnica es el embudo de marketing, el cuallogra detectar los clientes altamente potenciales para el negocio (Kotler \& Armstrong, 2012).

El comportamiento del consumidor influye mucho en su decisión de compra, por lo tanto, es normal que las empresas se preocupen por estimular ese deseo de compra mediante mejores opciones de precio, gama de productos, técnicas de información, métodos de pago y más (Santana, Reis, \& Oliveira, 2016). Los vendedores deben considerar aspectos económicos, tecnológicos, políticos y culturales a la hora de mostrar un nuevo producto al mercado y posicionarlo, pues cada segmento de este requerirá alguna de estas variables específicas (Henao \& Córdoba, 2007).

En otras palabras, el cliente busca que el producto o servicio no sólo sea algo que necesite sino que cuente con aspectos como calidad, mayor 
rendimiento y características innovadoras; es decir, busca una mejora continua de estas variables, las cuales constituyen partes importantes de la mayoría de las estrategias de marketing. La organización debe estar en búsqueda constante de satisfacer necesidades sin dejar de lado los objetivos que encierran su misión y un enfoque de comercialización, valor y atención al cliente (Espinoza, Campos, \& Fabiani, 2015).

En cuanto a una base legal que respalde estos procesos que se dan en los mercados tenemos la Ley Orgánica de Regulación y Control del Poder de Mercado, la cual rige dichos procesos en la República del Ecuador, y la Superintendencia de Control de Poder del Mercado de Ecuador, que es el ente encargado de regular y supervisar dichos procesos. Lo que se busca con la aplicación de estas leyes es evitar, prevenir, corregir, eliminar y sancionar el abuso de operadores económicos con poder de mercado y buscar la eficiencia de los mercados con la finalidad de que se vuelva un sistema económico, social, solidario y sostenible (Ley Orgànica de Regulaciòn y Control del Poder de Mercado, 2013).

\section{Metodología}

Para cumplir los objetivos del trabajo se utilizaron diferentes tipos de investigación, de los cuales se puede destacar la metodología empírico positivista porque utiliza la experiencia vivida para generar nuevo conocimiento científico. Por otra parte, se consideró la investigación de campo en relación a la muestra de estudio, tomando como población los mercados de la bahía de Guayaquil de la provincia del Guayas. También se empleó la Investigación Exploratoria, la cual pretende dar una visión general respecto a una realidad ybusca obtener información preliminar que ayude a definir problemas y sugerir hipótesis (Kotler \& Armstrong, 2008). De igual manera se tomó en cuenta la Investigación Descriptiva, la cual analiza de manera detallada los resultados del estudio ya que implica técnicas como la observación y posterior descripción del sujeto de estudio sin distorsionar la realidad (Behar, 2008). Finalmente fue también puesta en prácticala Investigación Cuantitativa, la cual recoge la información para posteriormente probar las hipótesis mediantes modelos estadísticos y medición cuantificable con el fin de comprobar teorías y las hipótesis planteadas. Utiliza también parte de la literatura y los objetivos del estudio (Sampieri, Collado, \& Lucio, 2014).

El mercado de la Bahía es muy extenso, tiene aproximadamente unos 7000 negocios. A efectos de esta situación, en donde el universo de la población es muy grande, se tomó como población los negocios correspondientes al sector que comprende las calles. Ayacucho y Eloy Alfaro las cualeshacen un total de 95 puestos de trabajo que son la nueva población en relación a las calles que se escogieron. Como bien se ha mencionado, la población está conformada por los negocios situados en la bahía de Guayaquil 
donde se tomó una muestra por conveniencia. Este tipo de muestreo no probabilístico permite que los sujetos sean seleccionados dada la conveniente accesibilidad y proximidad de los sujetos para el investigador. Estonos dio un total de 76 negocios que fueron encuestados, lo que corresponde al $80 \%$ del total de la población correspondiente a los 95 negocios ya mencionados.

Tabla 2. Ficha Técnica

\begin{tabular}{|l|l|}
\hline Delimitación Geográfica & Ecuador \\
\hline Provincia & Guayas \\
\hline Cantón & Guayaquil \\
\hline Universo & Negocios de la Bahía de Guayaquil \\
\hline Muestreo & $\begin{array}{l}\text { Se utilizó parte de la población escogida de un estrato del } \\
\text { universo en donde la muestra arrojó un total de 76 personas a } \\
\text { encuestar. }\end{array}$ \\
\hline Instrumento de & $\checkmark \quad$ Encuesta \\
Recolección de datos & $\checkmark \quad$ Observación \\
\hline Población & 95 negocios de la Bahía ubicados en las calles: Ayacucho y Eloy \\
& Alfaro \\
\hline Fecha de realización & Agosto - Septiembre 2017 \\
\hline Programa estadístico & IBM SPSS versión 22 \\
\hline
\end{tabular}

\section{Elaboración propia}

Ejemplo de cálculo

$$
\begin{aligned}
& n \frac{Z^{2} N P Q}{e^{2} N+Z^{2} P Q} \\
& n \frac{(1,96)^{2}(95)(0,5)(0,5)}{(0,05)^{2}(95)+(1,96)^{2}(0,5)(0,5)}=76
\end{aligned}
$$

Como instrumentos se han escogido las técnicas de la encuesta y la observación,técnicas que permiten la recolección de datos que posteriormente pueden ser interpretados y generar un nuevo conocimiento. En el caso de la encuesta la recolección de datos, al ser cuantitativa, se somete bajo programas estadísticos, en este caso el IBM SPSS versión 22, el cual hace más sencilla la tabulación y análisis de las variables. Con respecto a la encuesta, se elaboraron 7 preguntas cerradas dicotómicas con las que se trataba de conseguir una respuesta directa hacia las variables planteadas, 2 preguntas de escala de Likert con la finalidad de conocer el grado de satisfacción de los encuestados con respecto a lo que se propone y 1 pregunta de conocimiento. La encuesta también ha permitido obtener diversos tipos información; es el método más utilizado para recabar datos primarios y es el enfoque idóneo para obtener información descriptiva (Kotler \& Armstrong, 2008). 


\section{Resultados}

Tabla 3. El significado de Estrategia de Marketing y Segmentación de Mercado

\begin{tabular}{|c|c|c|c|c|c|}
\hline & & Frecuencia & Porcentaje & $\begin{array}{l}\text { Porcentaje } \\
\text { válido }\end{array}$ & $\begin{array}{l}\text { Porcentaje } \\
\text { acumulado }\end{array}$ \\
\hline \multirow[t]{3}{*}{ Válido } & $\mathrm{Si}$ & 37 & 48,1 & 48,7 & 48,7 \\
\hline & No & 39 & 50,6 & 51,3 & 100,0 \\
\hline & Total & 76 & 98,7 & 100,0 & \\
\hline Perdidos & Sistema & 1 & 1,3 & & \\
\hline Total & & 77 & 100,0 & & \\
\hline
\end{tabular}

Fuente: Guayaquil - encuesta realizada de Agosto- Septiembre del 2017.

Elaboración propia

En esta tabla se muestra que el menor porcentaje relacionado a la pregunta arroja que los encuestados respondieron que sí, dado a que, al menos, conocían algo acerca de lo que es una estrategia de marketing pero de forma muy general, pues ellos entienden al marketing como una forma de enganchar al cliente con su producto o servicio. Por el contrario, un mayor porcentaje de los encuestados respondieron que no conocían nada acerca de la variable abordada en la misma.

Tabla 4. Una segmentación de los mercados de la Bahía de Guayaquil, reduce el índice de insatisfacción de los clientes.

\begin{tabular}{|c|c|c|c|c|c|}
\hline & & Frecuencia & Porcentaje & $\begin{array}{l}\text { Porcentaje } \\
\text { válido }\end{array}$ & $\begin{array}{l}\text { Porcentaje } \\
\text { acumulado }\end{array}$ \\
\hline \multirow[t]{3}{*}{ Válido } & $\mathrm{Si}$ & 48 & 62,3 & 63,2 & 63,2 \\
\hline & No & 28 & 36,4 & 36,8 & 100,0 \\
\hline & Total & 76 & 98,7 & 100,0 & \\
\hline Perdidos & Sistema & 1 & 1,3 & & \\
\hline Total & & 77 & 100,0 & & \\
\hline
\end{tabular}

Fuente: Guayaquil - encuesta realizada de Agosto- Septiembre del 2017.

Elaboración propia

Se puede apreciar que el 36,84\% de los negocios encuestados no están de acuerdopor el hecho de que una implementación de la segmentación de mercado en los negocios de la bahía de Guayaquil no ayudaría a que el índice de insatisfacción de los clientes se redujera. Por otro lado, el otro $63,16 \%$ está de acuerdo porquepiensan que si se implementa una estrategia de marketing en la bahía de Guayaquil como lo es la segmentación de los negocios, esto podría ayudar a que los clientes tengan una mayor satisfacción al momento de adquirir un producto. 
Tabla 5. Los factores que impiden la aplicación de una segmentación de mercados en los negocios comerciales de la bahía de Guayaquil.

\begin{tabular}{|c|c|c|c|c|c|}
\hline & & Frecuencia & Porcentaje & $\begin{array}{l}\text { Porcentaje } \\
\text { válido }\end{array}$ & $\begin{array}{l}\text { Porcentaje } \\
\text { acumulado }\end{array}$ \\
\hline \multirow[t]{4}{*}{ Válido } & $\begin{array}{l}\text { Factor } \\
\text { conocimiento }\end{array}$ & 22 & 28,6 & 28,9 & 28,9 \\
\hline & $\begin{array}{l}\text { Factor } \\
\text { resistencia al } \\
\text { cambio }\end{array}$ & 27 & 35,1 & 35,5 & 64,5 \\
\hline & $\begin{array}{l}\text { Factor } \\
\text { económico }\end{array}$ & 27 & 35,1 & 35,5 & 100,0 \\
\hline & Total & 76 & 98,7 & 100,0 & \\
\hline Perdidos & Sistema & 1 & 1,3 & & \\
\hline Total & & 77 & 100,0 & & \\
\hline
\end{tabular}

Fuente: Guayaquil - encuesta realizada de Agosto- Septiembre del 2017. Elaboración propia

Los resultados demuestran que los negocios de la bahía de Guayaquil no implementan esta estrategia de marketing por varios factores, siendo los de mayor porcentajelos factores económicos con un porcentaje de $35,53 \%$ y el factor resistencia al cambio con el 35,53\%, esto porque los vendedores quizás temenimplementar nuevas estrategias por la inversión que estas demandan para ejecutarla o simplemente tienen miedo al fracaso por el modelo de venta que han llevado por años. El de menor importancia fue el factor conocimiento que está representado por $28,95 \%$.

Tabla 6. Falta de organización impide dar un mejor servicio o atención al cliente.

\begin{tabular}{|c|c|c|c|c|c|}
\hline & & Frecuencia & Porcentaje & $\begin{array}{l}\text { Porcentaje } \\
\text { válido }\end{array}$ & $\begin{array}{l}\text { Porcentaje } \\
\text { acumulado }\end{array}$ \\
\hline \multirow[t]{3}{*}{ Válido } & $\mathrm{Si}$ & 49 & 63,6 & 64,5 & 64,5 \\
\hline & No & 27 & 35,1 & 35,5 & 100,0 \\
\hline & Total & 76 & 98,7 & 100,0 & \\
\hline Perdidos & Sistema & 1 & 1,3 & & \\
\hline Total & & 77 & 100,0 & & \\
\hline
\end{tabular}

Fuente: Guayaquil - encuesta realizada de Agosto- Septiembre del 2017.

Elaboración propia

En este caso la mayoría de los puestos encuestados de la Bahía de Guayaquil consideran quela falta de organización impide una buena atención a los clientes y por ende la localización de los productos de forma efectiva. La otra parte que representaun 35,53\% afirma que para ellos la buena atención de los clientes no se ve afectada por la organización de los negocios de la bahía de Guayaquil. 


\section{Discusión}

Es muy importante conocer que cada una de las variables implementadas en la encuesta permitió demostrar las hipótesis planteadas. Por tal razón, se procedió a utilizar la correlación de las variables en donde podemos percatarnos que las persona encuestadas de la Bahía de Guayaquil conocen sobre ¿Qué es una estrategia de marketing?, pero de una forma general, mas sin embargo desconocen el potencial de las ventajas que representan estos procesos del marketing. En cuanto a la Segmentación de Mercado levemente entendían su significado. De igual manera, la muestra encuestada reconoció que hay factores que no permitirían la aplicación de una segmentación de mercado, ejemplo de ello son el factor económico y el de resistencia al cambio. En cuanto a las otras variables, la organización que mantienen estos negocios en la bahía es regular, lo cual también influye en la poca insatisfacción del cliente. A pesar de todo lo mencionado, los comerciantes creen que una segmentación de mercado sí sería una estrategia del marketing que permitiría a sus negocios servistos con una mejor distribución y fácil acceso a lo que ofrecen, lográndose una competencia justa.

Tabla 7. Pruebas De Chi-Cuadrado de la variable Conocimiento del significado de estrategias de marketing y segmentación de mercado entre la variable Factores que impiden la aplicación de una segmentación de mercado

\begin{tabular}{l|l|l|l|l|l}
\hline & Valor & gl & $\begin{array}{l}\text { Sigasintótica (2 } \\
\text { caras) }\end{array}$ & $\begin{array}{l}\text { Significación } \\
\text { exacta }\end{array}$ & $\begin{array}{l}\text { Significación } \\
\text { exacta }\end{array}$ \\
\hline $\begin{array}{l}\text { Chi-cuadrado } \\
\text { de Pearson }\end{array}$ & $71,790^{\mathrm{a}}$ & 1 &, 000 & & \\
\hline $\begin{array}{l}\text { Corrección de } \\
\text { continuidad } \\
\text { Razón de } \\
\text { verosimilitud }\end{array}$ & 67,642 & 1 &, 000 &, 000 &, 000 \\
\hline $\begin{array}{l}\text { Prueba exacta } \\
\text { de Fisher }\end{array}$ & 90,270 & 1 &, 000 & & \\
\hline $\begin{array}{l}\text { Asociación } \\
\text { lineal por lineal }\end{array}$ & 70,845 & 1 &, 000 & & \\
\hline $\begin{array}{l}\text { N de casos } \\
\text { válidos }\end{array}$ & 76 & & & & \\
\hline $\begin{array}{l}\text { a. } 0 \text { casillas }(0,0 \%) \\
9,95 .\end{array}$ \\
\begin{tabular}{l} 
- Sólo se ha calculado para una tabla 2x2 \\
\hline
\end{tabular}
\end{tabular}

Fuente: Guayaquil - encuesta realizada de Agosto- Septiembre del 2017.

Elaboración propia

Efectivamente, la hipótesis planteada es verdadera, ya que el conocimiento de estrategias de marketing está relacionado a factores como conocimiento, economía y resistencia al cambio, que de una u otra forma influyen en la implementación de una segmentación de mercado. Las variables 
planteadas en las pregunta 2 y 6 son dependientes, puesto que los comerciantes de la bahía de Guayaquil tienen previos conocimientos acerca de los que son estrategias de ventas. El factor que más influye a la hora de preguntar qué impide una implementación de segmentación de mercado como estrategia de marketing es el factor económico y el factor resistencia al cambio.

Tabla 8. Pruebas De Chi-Cuadrado de la variable Insatisfacción de los clientes entre la variable Falta de organización de los negocios de la bahía de Guayaquil

\begin{tabular}{l|l|l|l}
\hline & Valor & gl & Sig. asintótica (2 caras) \\
\hline Chi-cuadrado de Pearson & $49,315^{\mathrm{a}}$ & 2 &, 000 \\
\hline Razón de verosimilitud & 68,210 & 2 &, 000 \\
\hline Asociación lineal por lineal & 48,455 & 1 &, 000 \\
\hline N de casos válidos & 76 & & \\
\hline $\begin{array}{l}\text { a. } 0 \text { casillas (0,0\%) han esperado un recuento menor que 5. El recuento mínimo esperado } \\
\text { es 10,71. }\end{array}$
\end{tabular}

Fuente: Guayaquil - encuesta realizada de Agosto- Septiembre del 2017. Elaboración propia

Ciertamente, la segunda hipótesis planteada es verdadera, ya que la variable de la pregunta 4 tiene relación con la variable de la pregunta 7; estas dos variables son dependientes de acuerdo a los resultados estadísticos arrojados por el programa utilizado, lo que quiere decir que de una u otra forma la atención a los clientes influye en la satisfacción de ellos.

\section{Conclusión}

A partir de los resultados de la investigación se planteaque los dueños de los negocios de la Bahía de Guayaquil conocen términos como Marketing y estrategias, pero cuando nos referimosa segmentación de mercado no tienen un claro concepto del mismo. Sin embargo, con el simple hecho de saber que es una de las estrategias de marketing, los mismos consideran que su aplicación ayudaría a la organización de sus negocios, permitiendo que los bienes y servicios que ofrecen tengan una mejor agrupación y visualización ante el cliente y conlleven por ende a su satisfacción al adquirirlo, evitando además que el mismo cliente se moleste por el hecho de no encontrar lo que busca. En resumen, los comerciantes ven a la segmentación como un proceso eficaz y efectivo a la hora de distribuir los negocios y generar una competencia igual entre los vendedores.

De igual manera, existe el temor en los comerciantes a la hora de implementarla, pues creen que no cuentan con las facilidades o la seguridad que se requieren para poder hacer uso de dicha estrategia, hecho quese pudo evidenciar en los factores económicos y de resistencia al cambio. Se podría establecer que en lo que respecta al factor económico existe el temor a invertir en algo desconocido y poco aplicado en el país, y en cuanto al factor resistencia al cambio se puede plantear que ellos están acostumbrados a un 
sistema de venta llevado por años y a su manera, sin duda con ciertas reglas impuestas por leyes vigentes, pero para ellos este proceso de la segmentación de mercado es en verdad algo nuevo y desconocido.

Como aporte de política pública para este trabajo tenemos la Ley Orgánica de Regulación y Control de poder de Mercado la cual en su Art. 1 hace referencia sobre el objetivo de su existencia en los mercados del comercio y el fin que se busca en la economía de la sociedad ecuatoriana. Mediante estas leyes se trata de reorganizar el mercado y poner limitantes de lo que se debe hacer y evitar. De igual modo, promueve una competencia justa y equitativa entre todos los comerciantes de dichos sectores. Dentro de sus disposiciones también se encuentra la ayuda que deben brindar estos organismos en la implementación de medidas o estrategias que permitan al comerciante contar con las condiciones para poder satisfacer las necesidades del cliente. Existen muchos artículos dentro de esta Ley que promueven un sinnúmero de acciones que permiten mejorar la eficiencia y eficacia de dichos mercados.

Se propone también hacer un plan piloto donde el G.A.D Municipal de Guayaquil de la Provincia de Guayas, intervenga en la organización de los negocios de la Bahía e invierta en nuevas estrategias de marketing en este caso, la aplicación de una segmentación de mercado en dichos negocios, a fin de que se reestructuren dichas plazas de comercio y se logre la eficiencia por medio de la organización de los negocios y la eficacia al satisfacer las necesidad del cliente.

\section{References:}

1. Armstrong, G., \& kotler, P. (2013). Fundamentos de Marketing (11 ed.). (G. D. Chávez, Ed.) Mexico: Pearson Educación. Recuperado el 26 de Agosto de 2017, de http://librospdfgratis.org/index.php/2015/12/16/fundamentos-demarketing-11-edicion-kotler-armstrong-pdf/

2. Arrieta, A. J. (2007). El valor del cliente como herramienta estratégica de gestión en un mercado industrial. Tesis Doctoral, 402. Màlaga, España: Departamento de Economia y Administracion de Empresas de la Universidad de Màlaga . Recuperado el 30 de Agosto de 2017, de http://www.biblioteca.uma.es/bbldoc/tesisuma/17114111.pdf

3. Behar, D. (2008). Metodologia de la Investigacion (1 ed.). Editorial Shalom. Recuperado el 27 de Agosto de 2017, de http://www.trabajosocialbadajoz.es/colegio/wpcontent/uploads/2011/05/Intriducci\%C3\%B3n-a-laMetodolog\%C3\%ADa-de-la-Investigaci\%C3\%B3n.pdf

4. Cabrera, J. (2016). UPANAMA. Recuperado el 09 de Julio de 2017, de Universidad Panameña: http://www.upana.edu.gt/noticias/laimportancia-de-la-segmentacion-de-mercados/ 
5. Circulos de Lectores. (2004). Enciclopedia Práctica de Administración. Micro, Pequeña y Mediana Empresa (Vol. Tomo 1 ). Bogota, Colombia : Ediciones Credimar. Recuperado el 25 de Agosto de 2017

6. Ciribeli, J. P., \& Miquelito, S. (Junio de 2015). La segmentación del mercado por el criterio psicográfico: Un ensayo teórico sobre los principales enfoques psicográficos y su relación con los criterios de comportamiento. Vision de Futuro, Año 12, 119(1), 33 - 50. Recuperado el 28 de Agosto de 2017, de https://dialnet.unirioja.es/ejemplar/407842

7. Espinoza, R., Campos, H., \& Fabiani, B. (Junio de 2015). Arazá marketing and its influence on the economy of families the province of simon bolivar city Guayas. International Journal of Information Research and Review, 2(6), 808 - 813 . Recuperado el 30 de Agosto de 2017, de http://www.ijirr.com/araz\%C3\%A1-marketing-and-itsinfluence-economy-families-province-simon-bolivar-city-guayas

8. Guadarrama, E., \& Rosales, E. (2015). Marketing Relacional: Valor, Satisfacción, Lealtad y Retención del Cliente. Análisis y Retención Teórica. Ciencia y Sociedad, 40 (2), 307- 340 . Recuperado el 27 de Agosto de 2017, de http://www.redalyc.org/articulo.oa?id=87041161004

9. Henao, O., \& Córdoba, J. (Diciembre de 2007). Comportamiento del consumidor, una mirada sociològica. Entramado, 3(2), 18- 29. Recuperado el 30 de Agosto de 2017, de www.redalyc.org/pdf/2654/265420387003.pdf

10. Keller, P., \& Kotler, K. (2012). Dirección de Marketing (14ª edición ed.). Mexico: Pearson Educaciòn.

11. Kotler \& Armstrong. (2008). Fundamentos de Marketing (8 ediciòn ed.). Mexico, Naucalpan de Juárez: Pearson Educacion. Recuperado el 24 de Agosto de 2017, de https://utecno.files.wordpress.com/2016/09/kottler-phillip-armstronggary-fundamentos-de-marketing.pdf

12. Kotler, \& Armstrong. (2007). Marketing Version para Latinoamerica (11 Ediciòn ed.). (P. M. Rosas, Ed.) Mexico, Mexico: Pearson Education,. Recuperado el 27 de Agosto de 2017, de https://uvgcancun.files.wordpress.com/2016/03/kottler-philliparmstrong-gary-marketing-versic3b3n-para-latinoamc3a9rica.pdf

13. Kotler, P., \& Armstrong, G. (2012). Marketing (14 Ediciòn ed.). (G. D. Chávez, Ed.) Mèxico : Pearson Educación de México. Recuperado el 30 de Agosto de 2017, de https://profdariomarketing.files.wordpress.com/2014/03/marketing_k otler-armstrong.pdf 
14. Mullins, J., Walker, O., Boyd, H., \& Jamieson, B. (2013). Marketing Management ( 7 Ediciòn ed.). Reino Unido : The McGraw-Hill Companies Education. Recuperado el 28 de Agosto de 2017, de https://www.ebsglobal.net/documents/course-tasters/spanish/pdf/mkbk-taster.pdf

15. París, J. (Diciembre de 2013). Hacia la segmentación significativa. POLIANTEA, $\quad 9(17), \quad 11 \quad 39$. doi:http://dx.doi.org/10.15765/plnt.v9i17.462

16. Robín, C. F., \& Torres, C. A. (Junio de 2001). Segmentación de mercados: buscando la correlación entre variables sicológicas y demográficas. Colombiana de Marketing, 2(2), 1-14. Recuperado el 29 de Agosto de 2017, de http://www.redalyc.org/pdf/109/10900204.pdf

17. Sampieri, R. H., Collado, C. F., \& Lucio, M. B. (2014). Metodologia de la Investigaciòn (6 Ediciòn ed.). (M. Á. Castellanos, Ed.) Mexico: McGRAW-HILL / INTERAMERICANA EDITORES, S.A. Recuperado el 29 de Agosto de 2017 , de https://metodologiaecs.wordpress.com/2016/01/31/libro-metodologiade-la-investigacion-6ta-edicion-sampieri-pdf/

18. Santana, D. O., Reis, A. C., \& Oliveira, R. S. (2016). Los estudios del comportamiento del consumidor en SciELO. REVISTA DE LA FACHE, 5(2), 1- 16. Recuperado el 30 de Agosto de 2017, de http://www.palabraclave.fahce.unlp.edu.ar/article/view/PCv5n2a03

19. Silk, J. (2006). Que es Marketing (1 Edicion ed.). São Paulo. Recuperado el 24 de Agosto de 2017.

20. Smith, W. R. (Julio de 1956). Product Differentiation and Market Segmentation as Alternative Marketing Strategies. Journal of Marketing, 21(1), 3-8. doi:10.2307/1247695

21. Stanton, W. J., Etze, M. J., \& Walker, B. J. (2007 ). Fundamentos de Marketing (14 Ediciòn ed.). (R. A. Alayón, Ed.) Mèxico : McGRAWHILL/INTERAMERICANA EDITORES. Recuperado el 30 de Agosto de

2017,dehttps://mercadeo1marthasandino.files.wordpress.com/2015/02 /fundamentos-de-marketing-stanton-14edi.pdf 